\title{
Analysis of a Discontinuous Finite Element Method for the Coupled Stokes and Darcy Problems
}

\author{
Béatrice Rivière ${ }^{1}$
}

Received July 28, 2003; accepted (in revised form) May 5, 2004

The coupled Stokes and Darcy flows problem is solved by the locally conservative discontinuous Galerkin method. Optimal error estimates for the fluid velocity and pressure are derived.

KEY WORDS: Surface and subsurface flow; error estimates; interface conditions

\section{INTRODUCTION}

Modeling the interaction between surface and subsurface flow is a challenging environmental problem. One such example is the simulation of transport of contaminants through rivers into the aquifers. Mathematically, this complex problem can be modeled by the coupled system of Stokes and Darcy equations.

The aim of this paper is to formulate and analyze a discontinuous finite element method for the coupled Stokes and Darcy problems. The physical domain is decomposed into two regions: the region filled with an incompressible fluid modeled by the Stokes equations and the porous medium region modeled by Darcy's law. The interface conditions consist of the Beavers-Joseph-Saffman condition, the continuity of flux and the balance of forces. The matching condition of meshes at the interface can be relaxed. The unknowns, namely the fluid velocity and pressure in the fluid region, and the fluid pressure in the porous medium, are approximated by totally discontinuous polynomials of different order. The discontinuous Galerkin (DG) methods considered here, are based on the

\footnotetext{
${ }^{1} 301$ Thackeray, University of Pittsburgh, Pittsburgh, PA 15260, USA. E-mail: riviere@math.pitt.edu.
} 


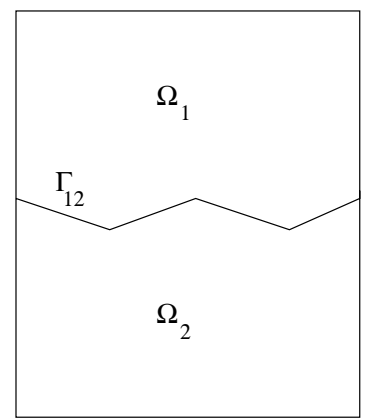

Fig. 1. Example of domain.

Non-symmetric Interior Penalty Galerkin (NIPG) method $[18,19]$ and the Symmetric Interior Penalty Galerkin (SIPG) method [2]. DG methods are attractive methods because they are element-wise conservative, they are high-order methods, and they are easily implementable on unstructured meshes. A few algorithms for the coupling of Stokes and Darcy can be found in the literature. In a paper by Layton et al. [15], the existence and uniqueness of a weak solution to the coupled system is proved and the proposed scheme combines the continuous finite element method for the Stokes problem with the mixed finite element method for the Darcy equations. The study of continuous finite element methods for both regions can be found in the work of Discacciati et al. [8,9]. In a more recent work [20], the DG method is used in the Stokes region while the mixed finite element method is used for Darcy region. Finally, the reader can refer to $[4,10,16$, 17] for analysis of similar coupled models.

The outline of the paper is as follows. Section 2 contains the model problem and notation. The numerical scheme is introduced in Sec. 3. The a priori error estimates are proved in Sec. 4 and followed by some concluding remarks.

\section{MODEL PROBLEM AND NOTATION}

Let $\Omega$ be a polygonal domain in $\mathbb{R}^{2}$, subdivided into two subdomains $\Omega_{1}, \Omega_{2}$, with interface $\Gamma_{12}=\partial \Omega_{1} \cap \partial \Omega_{2}$ (see Fig. 1). Define $\Gamma_{i}=\partial \Omega_{i}-\Gamma_{12}$ for $i=1,2$. The physical quantities are the fluid velocity $\boldsymbol{u}$ and pressure $p$. Denote $\boldsymbol{u}_{i}=\left.\boldsymbol{u}\right|_{\Omega_{i}}$ and $p_{i}=\left.p\right|_{\Omega_{i}}$. We assume that the flow satisfies the Stokes equations on $\Omega_{1}$ and the single phase equations on $\Omega_{2}$.

$$
-\nabla \cdot\left(2 \mu \boldsymbol{D}\left(\boldsymbol{u}_{1}\right)-p_{1} \boldsymbol{I}\right)=\boldsymbol{f}_{1} \quad \text { in } \quad \Omega_{1},
$$




$$
\begin{array}{rcc}
\nabla \cdot \boldsymbol{u}_{1}=0 & \text { in } & \Omega_{1}, \\
\boldsymbol{u}_{2}=-\boldsymbol{K} \nabla p_{2} & \text { in } & \Omega_{2}, \\
\nabla \cdot \boldsymbol{u}_{2}=f_{2} & \text { in } & \Omega_{2} .
\end{array}
$$

Here, $\boldsymbol{f}_{1}$ and $f_{2}$ are external forces acting on the fluid, $\mu>0$ is the constant fluid viscosity, $\boldsymbol{D}(\boldsymbol{u})$ is the strain tensor defined by $\boldsymbol{D}(\boldsymbol{u})=$ $(1 / 2)\left(\nabla \boldsymbol{u}+\nabla \boldsymbol{u}^{T}\right)$ and $\boldsymbol{K}$ is the permeability tensor. We assume that $\boldsymbol{K}$ is symmetric, positive definite tensor, bounded below and above uniformly, and that the force satisfies the solvability condition $\int_{\Omega_{2}} f_{2}=0$. The boundary conditions are

$$
\begin{aligned}
\boldsymbol{u}_{1}=\mathbf{0} & \text { on } \quad \Gamma_{1}, \\
-\boldsymbol{K} \nabla p_{2} \cdot \boldsymbol{n} & =0 \quad \text { on } \quad \Gamma_{2},
\end{aligned}
$$

where $\boldsymbol{n}$ is the outward normal to the boundary $\partial \Omega$. As the pressure is unique up to an additive constant, we assume that

$$
\int_{\Omega} p=0 .
$$

Let $\boldsymbol{n}_{12}$ (respectively $\boldsymbol{\tau}_{12}$ ) be the unit normal (respectively tangential) vector to $\Gamma_{12}$ outward of $\Omega_{1}$. The conditions at the interface $\Gamma$ are

$$
\begin{array}{r}
\boldsymbol{u}_{1} \cdot \boldsymbol{n}_{12}=\boldsymbol{u}_{2} \cdot \boldsymbol{n}_{12}, \\
p_{1}-2 \mu\left(\boldsymbol{D}\left(\boldsymbol{u}_{1}\right) \boldsymbol{n}_{12}\right) \cdot \boldsymbol{n}_{12}=p_{2}, \\
\boldsymbol{u}_{1} \cdot \boldsymbol{\tau}_{12}=-2 G\left(\boldsymbol{D}\left(\boldsymbol{u}_{1}\right) \boldsymbol{n}_{12}\right) \cdot \boldsymbol{\tau}_{12} .
\end{array}
$$

Interface conditions (8) and (9) represent the mass conservation and the balance of forces, respectively, across the interface. The Beaver-JosephSaffman law (10) is the most accepted condition [3,14,21] and includes a friction constant $G>0$ that can be determined experimentally. The existence of a unique weak solution of (1)-(10) was shown in [15]. We assume here that the solution $(\boldsymbol{u}, p)$ is regular enough, and is a strong solution of (1)-(10).

We now define the functional spaces. For $i=1,2$, let $\mathcal{E}_{h}^{i}$ be a nondegenerate quasi-uniform subdivision of $\Omega_{i}$, let $\Gamma_{h}^{i}$ be the set of interior edges and let $h_{i}$ denote the maximum diameter of elements in $\mathcal{E}_{h}^{i}$. We assume that the meshes at the interface are non-matching in the following sense: any edge $e=\partial E^{1} \cap \Gamma_{12}$ where $E^{1}$ belongs to $\mathcal{E}_{h}^{1}$, belongs to one element $E^{2} \in \mathcal{E}_{h}^{2}$ and one only. For any non-negative integer $k$ and number $r \geqslant 1$, the classical Sobolev space [1] on a domain $\mathcal{O}$ is denoted by $W^{k, r}(\mathcal{O})=\left\{v \in L^{r}(\mathcal{O}): D^{m} v \in L^{r}(\mathcal{O}), \quad \forall|m| \leqslant k\right\}$, where 
$D^{m} v$ are the partial derivatives of $v$ of order $m$. The associated Sobolev norm (respectively, semi-norm) is denoted by $\|\cdot\|_{k, r, \mathcal{O}}$ (respectively, $|\cdot|_{k, r, \mathcal{O}}$ ), or by $\|\cdot\|_{k, \mathcal{O}}$ (respectively, $\left.|\cdot|_{k, \mathcal{O}}\right)$ if $r=2$. We use the usual notation $H^{k}(\mathcal{O})$ for $W^{k, 2}(\mathcal{O})$ and $L_{0}^{2}(\mathcal{O})$ for the space of squareintegrable functions with zero average. The $L^{2}$ inner-product will be denoted by $(\cdot, \cdot)$. Throughout the paper, $C$ will denote a generic positive constant whose value may vary but will be independent of the mesh sizes $h_{1}$ and $h_{2}$. Our scheme requires that the trace of $p_{1}$ and the trace of the normal derivatives of $\boldsymbol{u}_{1}$ and $p_{2}$ are well defined, and are square-integrable. Therefore, we define the following functional spaces:

$$
\begin{array}{ll}
\boldsymbol{X}^{1}=\left\{\boldsymbol{v}_{1} \in\left(L^{2}\left(\Omega_{1}\right)\right)^{2}:\right. & \left.\forall E \in \mathcal{E}_{h}^{1},\left.\quad \boldsymbol{v}_{1}\right|_{E} \in\left(W^{2,4 / 3}(E)\right)^{2}\right\}, \\
M^{1}=\left\{q_{1} \in L^{2}\left(\Omega_{1}\right):\right. & \left.\forall E \in \mathcal{E}_{h}^{1},\left.\quad q_{1}\right|_{E} \in W^{1,4 / 3}(E)\right\}, \\
M^{2}=\left\{q_{2} \in L^{2}\left(\Omega_{2}\right):\right. & \left.\forall E \in \mathcal{E}_{h}^{2},\left.\quad q_{2}\right|_{E} \in W^{2,2}(E)\right\} .
\end{array}
$$

We associate to these spaces the following norms $\|\cdot\|_{X^{1}},\|\cdot\|_{M^{1}}$ and seminorm $\|\cdot\|_{M^{2}}$ :

$$
\begin{gathered}
\left\|\boldsymbol{v}_{1}\right\|_{X^{1}}^{2}=\left\|\nabla \boldsymbol{v}_{1}\right\|_{0, \Omega_{1}}^{2}+\sum_{e \in \Gamma_{h}^{1} \cup \Gamma_{1}} \frac{\sigma_{1, e}}{|e|}\left\|\left[\boldsymbol{v}_{1}\right]\right\|_{0, e}^{2}+\frac{1}{G} \sum_{e \in \Gamma_{12}}\left\|\boldsymbol{v}_{1} \cdot \boldsymbol{\tau}_{12}\right\|_{0, e}^{2}, \\
\left\|q_{1}\right\|_{M^{1}}^{2}=\left\|q_{1}\right\|_{0, \Omega_{1}}^{2}, \\
\left\|q_{2}\right\|_{M^{2}}^{2}=\left\|\nabla q_{2}\right\|_{0, \Omega_{2}}^{2}+\sum_{e \in \Gamma_{h}^{2}} \frac{\sigma_{2, e}}{|e|}\left\|\left[q_{2}\right]\right\|_{0, e}^{2} .
\end{gathered}
$$

Here, $|e|$ denotes the measure of each edge $e$, the parameters $\sigma_{1, e}$ and $\sigma_{2, e}$ are positive constants that may vary on each edge, and that will be defined in Sec. 3. Finally, the $\|\cdot\| \|$ norm is the usual "broken" norm: $\|w\|_{m, \Omega_{i}}^{2}=\sum_{E \in \mathcal{E}_{h}^{i}}\|w\|_{m, E}^{2}$ for $i=1,2$ and for $w$ any scalar or vector function. Given a fixed normal vector $\boldsymbol{n}_{e}$ on each edge $e=\partial E_{e}^{1} \cap \partial E_{e}^{2}$, pointing from $E_{e}^{1}$ to $E_{e}^{2}$, the average $\{w\}$ and jump [w] of function $w$ are uniquely defined

$$
\begin{aligned}
\{w\}= & \frac{1}{2}\left(\left.w\right|_{E_{e}^{1}}\right)+\frac{1}{2}\left(\left.w\right|_{E_{e}^{2}}\right), \quad[w]=\left(\left.w\right|_{E_{e}^{1}}\right)-\left(\left.w\right|_{E_{e}^{2}}\right), \\
& \{w\}=\left.w\right|_{E_{e}^{1}}, \quad[w]=\left.w\right|_{E_{e}^{1}} .
\end{aligned}
$$

The aim of this paper is to formulate an algorithm that uses totally discontinuous approximating spaces. Let $k_{1}, k_{2}$ be two positive integers and let the finite-dimensional subspaces $\boldsymbol{X}_{h}^{1} \subset \boldsymbol{X}^{1}, M_{h}^{1} \subset M^{1}$ and $M_{h}^{2} \subset M^{2}$, with 
the induced norms, be defined as follows:

$$
\begin{array}{lll}
\boldsymbol{X}_{h}^{1}=\left\{\boldsymbol{v}_{1} \in \boldsymbol{X}^{1}:\right. & \forall E \in \mathcal{E}_{h}^{1}, & \left.\boldsymbol{v}_{1} \in\left(\mathbb{P}_{k_{1}}(E)\right)^{2}\right\}, \\
M_{h}^{1}=\left\{q_{1} \in M^{1}:\right. & \forall E \in \mathcal{E}_{h}^{1}, & \left.q_{1} \in \mathbb{P}_{k_{1}-1}(E)\right\}, \\
M_{h}^{2}=\left\{q_{2} \in M^{2}:\right. & \forall E \in \mathcal{E}_{h}^{2}, & \left.q_{2} \in \mathbb{P}_{k_{2}}(E)\right\} .
\end{array}
$$

We assume that the discrete spaces satisfy the optimal approximation properties. In particular, there exists an operator $R_{h} \in \mathcal{L}\left(H^{1}\left(\Omega_{1}\right)^{2} ; \boldsymbol{X}_{h}^{1}\right)$ such that for any $E \in \mathcal{E}_{h}^{1}$,

$$
\begin{aligned}
& \forall \boldsymbol{v} \in H^{1}\left(\Omega_{1}\right)^{2}, \quad \forall q_{1} \in \mathbb{P}_{k_{1}-1}(E), \quad \int_{E} q_{1} \nabla \cdot\left(R_{h}(\boldsymbol{v})-\boldsymbol{v}\right)=0, \\
& \forall \boldsymbol{v} \in H^{1}\left(\Omega_{1}\right)^{2}, \quad \forall e \in \Gamma_{h}^{1}, \quad \forall \boldsymbol{q}_{1} \in \mathbb{P}_{k_{1}-1}(e)^{2}, \quad \int_{e} \boldsymbol{q}_{1} \cdot\left[R_{h}(\boldsymbol{v})\right]=0, \\
& \forall \boldsymbol{v} \in H_{0}^{1}\left(\Omega_{1}\right)^{2}, \quad \forall e \in \partial \Omega_{1}, \quad \forall \boldsymbol{q}_{1} \in \mathbb{P}_{k_{1}-1}(e)^{2}, \quad \int_{e} \boldsymbol{q}_{1} \cdot R_{h}(\boldsymbol{v})=0, \\
& \forall \boldsymbol{v} \in H^{1}\left(\Omega_{1}\right)^{2}, \quad \forall e \in \Gamma_{h}^{1} \cup \Gamma^{1} \cup \Gamma_{12}, \quad \int_{e}\left(R_{h}(\boldsymbol{v})-\boldsymbol{v}\right)=0, \\
& \forall \boldsymbol{v} \in H_{0}^{1}\left(\Omega_{1}\right)^{2}, \quad\left\|\boldsymbol{v}-R_{h}(\boldsymbol{v})\right\|_{X^{1}} \leqslant C\left\|\nabla\left(\boldsymbol{v}-R_{h}(\boldsymbol{v})\right)\right\|_{0, \Omega_{1}}, \\
& \forall \boldsymbol{v} \in W^{s, t}\left(\Omega_{1}\right)^{2}, \forall s \in\left[1, k_{1}+1\right], m=0,1,\left|\boldsymbol{v}-R_{h}(\boldsymbol{v})\right|_{m, t, E} \leqslant C h_{E}^{s-m}|\boldsymbol{v}|_{s, t, \Delta_{E}},
\end{aligned}
$$

where $\Delta_{E}$ is a suitable macro-element containing $E$. Note that property (18) is an easy consequence of (17) (see Ref. [12]). There exists also an operator $r_{h} \in \mathcal{L}\left(L^{2}(\Omega) ; M_{h}^{1} \times M_{h}^{2}\right)$ such that for $s=k_{1}-1, k_{2}$ and for any $z \in L^{2}(\Omega) \cap H^{s+1}(\Omega)$

$$
\begin{array}{r}
\forall q \in \mathbb{P}_{s}(E), \int_{E} q\left(r_{h}(z)-z\right)=0, \quad \forall E \in \mathcal{E}_{h}^{1} \cup \mathcal{E}_{h}^{2}, \\
\left\|z-r_{h}(z)\right\|_{m, E} \leqslant C h_{E}^{s+1-m}|z|_{s+1, E}, \quad \forall E \in \mathcal{E}_{h}^{1} \cup \mathcal{E}_{h}^{2}, \quad m=0,1 .
\end{array}
$$

Note that the existence of $R_{h}$ for $k_{1}=1,2$ or 3 follows from the nonconforming elements of Crouzeix et al. [7,6] and Fortin et al. [11], and the operator $r_{h}$ is the well-known $L^{2}$ projection operator.

We recall a result proved in Ref. [12] that generalizes a Sobolev imbedding. For any real number $s \in[2, \infty)$, there is a constant $C$ independent of $h$ such that

$$
\forall \boldsymbol{v} \in \boldsymbol{X}_{h}^{1}, \quad\left\|\boldsymbol{v}_{1}\right\|_{L^{s}\left(\Omega_{1}\right)} \leqslant C\left\|\boldsymbol{v}_{1}\right\|_{X^{1}} .
$$


We now finish this section with some trace and inverse inequalities needed for the analysis. Let $E$ be a mesh element with diameter $h_{E}$. Then, there exists a constant $C$ independent of $h_{E}$ such that

$$
\begin{array}{r}
\forall \phi \in H^{1}(E), \quad \forall e \subset \partial E, \quad\|\phi\|_{0, e}^{2} \leqslant C\left(h_{E}^{-1}\|\phi\|_{0, E}^{2}+h_{E}|\phi|_{1, E}^{2}\right), \\
\forall \phi \in H^{2}(E), \quad \forall e \subset \partial E, \quad\left\|\nabla \phi \cdot \boldsymbol{n}_{e}\right\|_{0, e}^{2} \leqslant C\left(h_{E}^{-1}|\phi|_{1, E}^{2}+h_{E}|\phi|_{2, E}^{2}\right), \\
\forall \phi \in \mathbb{P}_{k}(E), \quad \forall e \subset \partial E, \quad\left\|\nabla \phi \cdot \boldsymbol{n}_{e}\right\|_{0, e} \leqslant C h_{E}^{-1 / 2}|\phi|_{1, E} .
\end{array}
$$

\section{SCHEME}

We introduce the following bilinear forms $a_{1}: \boldsymbol{X}^{1} \times \boldsymbol{X}^{1} \rightarrow \mathbb{R}, b: \boldsymbol{X}^{1} \times$ $M^{1} \rightarrow \mathbb{R}$ and $a_{2}: M^{2} \times M^{2} \rightarrow \mathbb{R}:$

$$
\begin{aligned}
a_{1}\left(\boldsymbol{u}_{1}, \boldsymbol{v}_{1}\right)= & 2 \sum_{E \in \mathcal{E}_{h}^{1}} \int_{E} \boldsymbol{D}\left(\boldsymbol{u}_{1}\right): \boldsymbol{D}\left(\boldsymbol{v}_{1}\right)+\sum_{e \in \Gamma_{h}^{1} \cup \Gamma_{1}} \frac{\sigma_{1, e}}{|e|} \int_{e}\left[\boldsymbol{u}_{1}\right] \cdot\left[\boldsymbol{v}_{1}\right] \\
- & 2 \sum_{e \in \Gamma_{h}^{1} \cup \Gamma_{1}} \int_{e}\left\{\boldsymbol{D}\left(\boldsymbol{u}_{1}\right) \boldsymbol{n}_{e}\right\} \cdot\left[\boldsymbol{v}_{1}\right]+2 \epsilon_{1} \sum_{e \in \Gamma_{h}^{1} \cup \Gamma_{1}} \int_{e}\left\{\boldsymbol{D}\left(\boldsymbol{v}_{1}\right) \boldsymbol{n}_{e}\right\} \cdot\left[\boldsymbol{u}_{1}\right] \\
+ & \frac{1}{G} \sum_{e \in \Gamma_{12}} \int_{e}\left(\boldsymbol{u}_{1} \cdot \boldsymbol{\tau}_{12}\right)\left(\boldsymbol{v}_{1} \cdot \boldsymbol{\tau}_{12}\right)+\sum_{e \in \Gamma_{1}} \int_{e} \boldsymbol{u}_{1} \cdot \boldsymbol{v}_{1}, \\
b_{1}\left(\boldsymbol{v}_{1}, p_{1}\right)= & -\sum_{E \in \mathcal{E}_{h}} \int_{E} p_{1} \nabla \cdot \boldsymbol{v}_{1}+\sum_{e \in \Gamma_{h}^{1} \cup \Gamma_{1}} \int_{e}\left\{p_{1}\right\}\left[\boldsymbol{v}_{1}\right] \cdot \boldsymbol{n}_{e}, \\
a_{2}\left(p_{2}, q_{2}\right)= & \sum_{E \in \mathcal{E}_{h}^{2}} \int_{E} \boldsymbol{K} \nabla p_{2} \cdot \nabla q_{2}+\sum_{e \in \Gamma_{h}^{2}} \frac{\sigma_{2, e}}{|e|} \int_{e}\left[p_{2}\right]\left[q_{2}\right] \\
& -\sum_{e \in \Gamma_{h}^{2}} \int_{e}\left\{\boldsymbol{K} \nabla p_{2} \cdot \boldsymbol{n}_{e}\right\}\left[q_{2}\right]+\epsilon_{2} \sum_{e \in \Gamma_{h}^{2}} \int_{e}\left\{\boldsymbol{K} \nabla q_{2} \cdot \boldsymbol{n}_{e}\right\}\left[p_{2}\right] .
\end{aligned}
$$

By introducing the parameters $\epsilon_{1}, \epsilon_{2}$ that take the value \pm 1 , we allow for non-symmetric or symmetric bilinear forms $a_{1}$ and $a_{2}$. Throughout the paper, we assume that:

Hypothesis A: we assume that the parameter $\sigma_{1, e}$ is bounded below by a sufficiently large positive value. For the parameter $\sigma_{2, e}$, if $\epsilon_{2}=1$, then one 
can choose $\sigma_{2, e}=1$, but if $\epsilon=-1, \sigma_{2, e}$ must be bounded below by a sufficiently large positive value.

The constraint on the parameters $\sigma_{1, e}$ and $\sigma_{2, e}$ is standard for the SIPG method, but here necessary for the NIPG (for $\sigma_{1, e}$ ) because of the generalized Korn's inequality (see Lemma 3.2).

We next define a bilinear form $\Lambda: M^{2} \times \boldsymbol{X}^{1} \rightarrow \mathbb{R}$ acting on the interface $\Gamma_{12}$.

$$
\Lambda\left(q_{2}, \boldsymbol{v}_{1}\right)=\sum_{e \in \Gamma_{12}} \int_{e} q_{2} \boldsymbol{v}_{1} \cdot \boldsymbol{n}_{12}, \quad \forall\left(q_{2}, \boldsymbol{v}_{1}\right) \in M^{2} \times \boldsymbol{X}^{1}
$$

With these forms, we propose the following variational problem of (1)-(10): Find $\left(\boldsymbol{u}_{1}, p_{1}, p_{2}\right) \in \boldsymbol{X}^{1} \times M^{1} \times M^{2}$, solution of

$$
\begin{array}{rr}
\mu a_{1}\left(\boldsymbol{u}_{1}, \boldsymbol{v}_{1}\right)+b\left(\boldsymbol{v}_{1}, p_{1}\right)+\Lambda\left(p_{2}, \boldsymbol{v}_{1}\right)=\left(\boldsymbol{f}_{1}, \boldsymbol{v}_{1}\right), & \forall \boldsymbol{v}_{1} \in X_{h}^{1}, \\
b\left(\boldsymbol{u}_{1}, q_{1}\right)=0, & \forall q_{1} \in M_{h}^{1}, \\
a_{2}\left(p_{2}, q_{2}\right)-\Lambda\left(q_{2}, \boldsymbol{u}_{1}\right)=\left(f_{2}, q_{2}\right), & \forall q_{2} \in M_{h}^{2}, \\
\int_{\Omega_{1}} p_{1}+\int_{\Omega_{2}} p_{2}=0 .
\end{array}
$$

Lemma 3.1. If $\left(\boldsymbol{u}_{1}, p_{1}, p_{2}\right)$ is the solution of the coupled Stokessingle phase flow problem (1)-(10), then $\left(\boldsymbol{u}_{1}, p_{1}, p_{2}\right)$ is the solution of (30)-(33).

Proof. Multiply the Stokes equation (1) by $v_{1}$, integrate by parts over one element $E \in \mathcal{E}_{h}^{1}$, and sum over all elements in $\mathcal{E}_{h}^{1}$

$$
\begin{aligned}
\sum_{E \in \mathcal{E}_{h}^{1}} & \int_{E}\left(-p_{1} \boldsymbol{I}+2 \mu \boldsymbol{D}\left(\boldsymbol{u}_{1}\right)\right): \nabla \boldsymbol{v}_{1}-\sum_{e \in \Gamma_{h}^{1}} \int_{e}\left[\left(-p_{1} \boldsymbol{I}+2 \mu \boldsymbol{D}\left(\boldsymbol{u}_{1}\right)\right) \boldsymbol{n}_{e} \cdot \boldsymbol{v}_{1}\right] \\
& -\sum_{e \in \Gamma_{12}} \int_{e}\left(-p_{1} \boldsymbol{I}+2 \mu \boldsymbol{D}\left(\boldsymbol{u}_{1}\right)\right) \boldsymbol{n}_{12} \cdot \boldsymbol{v}_{1}-\sum_{e \in \Gamma_{1}} \int_{e}\left(-p_{1} \boldsymbol{I}+2 \mu \boldsymbol{D}\left(\boldsymbol{u}_{1}\right)\right) \boldsymbol{n} \cdot \boldsymbol{v}_{1} \\
& =\int_{\Omega_{1}} \boldsymbol{f}_{1} \cdot \boldsymbol{v}_{1} .
\end{aligned}
$$


Noting that $\boldsymbol{D}\left(\boldsymbol{u}_{1}\right): \nabla \boldsymbol{v}_{1}=\boldsymbol{D}\left(\boldsymbol{u}_{1}\right): \boldsymbol{D}\left(\boldsymbol{v}_{1}\right)$ and $\boldsymbol{I}: \nabla \boldsymbol{v}_{1}=\nabla \cdot \boldsymbol{v}_{1}$, we can rewrite the equation

$$
\begin{aligned}
\sum_{E \in \mathcal{E}_{h}^{1}} & \int_{E}\left(2 \mu \boldsymbol{D}\left(\boldsymbol{u}_{1}\right): \boldsymbol{D}\left(\boldsymbol{v}_{1}\right)-p_{1} \nabla \cdot \boldsymbol{v}_{1}\right) \\
& -\sum_{e \in \Gamma_{h}^{1} \cup \Gamma_{1}} \int_{e}\left\{\left(-p_{1} \boldsymbol{I}+2 \mu \boldsymbol{D}\left(\boldsymbol{u}_{1}\right)\right) \boldsymbol{n}_{e}\right\} \cdot\left[\boldsymbol{v}_{1}\right] \\
& -\sum_{e \in \Gamma_{h}^{1}} \int_{e}\left[\left(-p_{1} \boldsymbol{I}+2 \mu \boldsymbol{D}\left(\boldsymbol{u}_{1}\right)\right) \boldsymbol{n}_{e}\right] \cdot\left\{\boldsymbol{v}_{1}\right\} \\
& -\sum_{e \in \Gamma_{12}} \int_{e}\left(-p_{1} \boldsymbol{I}+2 \mu \boldsymbol{D}\left(\boldsymbol{u}_{1}\right)\right) \boldsymbol{n}_{12} \cdot \boldsymbol{v}_{1} \\
& =\left(\boldsymbol{f}_{1}, \boldsymbol{v}_{1}\right) .
\end{aligned}
$$

By regularity of the true solution and with the boundary condition (5), we have

$$
\begin{aligned}
\sum_{E \in \mathcal{E}_{h}^{1}} & \int_{E}\left(2 \mu \boldsymbol{D}\left(\boldsymbol{u}_{1}\right): \boldsymbol{D}(\boldsymbol{v})-p_{1} \nabla \cdot \boldsymbol{v}_{1}\right)+\sum_{e \in \Gamma_{h}^{1} \cup \Gamma_{1}} \int_{e}\left\{p_{1}\right\}\left[\boldsymbol{v}_{1}\right] \cdot \boldsymbol{n}_{e} \\
& -2 \mu \sum_{e \in \Gamma_{h}^{1} \cup \Gamma^{1}} \int_{e}\left\{\boldsymbol{D}\left(\boldsymbol{u}_{1}\right) \boldsymbol{n}_{e}\right\} \cdot\left[\boldsymbol{v}_{1}\right]+2 \mu \epsilon_{1} \sum_{e \in \Gamma_{h}^{1} \cup \Gamma^{1}} \int_{e}\left\{\boldsymbol{D}\left(\boldsymbol{v}_{1}\right) \boldsymbol{n}_{e}\right\} \cdot\left[\boldsymbol{u}_{1}\right] \\
& -\sum_{e \in \Gamma_{12}} \int_{e}\left(-p_{1} \boldsymbol{I}+2 \mu \boldsymbol{D}\left(\boldsymbol{u}_{1}\right)\right) \boldsymbol{n}_{12} \cdot \boldsymbol{v}_{1}=\left(\boldsymbol{f}_{1}, \boldsymbol{v}_{1}\right)
\end{aligned}
$$

By decomposing $\boldsymbol{v}_{1}$ and $2 \mu \boldsymbol{D}\left(\boldsymbol{u}_{1}\right) \boldsymbol{n}_{12}$ into their normal and tangential components, the interface integral is reduced to

$$
\begin{aligned}
& -\sum_{e \in \Gamma_{12}} \int_{e}\left(-p_{1} \boldsymbol{I}+2 \mu \boldsymbol{D}\left(\boldsymbol{u}_{1}\right)\right) \boldsymbol{n}_{12} \cdot \boldsymbol{v}_{1} \\
= & \sum_{e \in \Gamma_{12}} \int_{e}\left(p_{1}-2 \mu\left(\boldsymbol{D}\left(\boldsymbol{u}_{1}\right) \boldsymbol{n}_{12}\right) \cdot \boldsymbol{n}_{12}\right)\left(\boldsymbol{v}_{1} \cdot \boldsymbol{n}_{12}\right) \\
& -\sum_{e \in \Gamma_{12}} \int_{e} 2 \mu\left(\boldsymbol{D}\left(\boldsymbol{u}_{1}\right) \boldsymbol{n}_{12}\right) \cdot \boldsymbol{\tau}_{12}\left(\boldsymbol{v}_{1} \cdot \boldsymbol{\tau}_{12}\right)
\end{aligned}
$$


With the interface conditions (9) and (10), the integral becomes

$$
\begin{gathered}
-\sum_{e \in \Gamma_{12}} \int_{e}\left(-p_{1} \boldsymbol{I}+2 \mu \boldsymbol{D}\left(\boldsymbol{u}_{1}\right)\right) \boldsymbol{n}_{12} \cdot \boldsymbol{v}_{1} \\
=\sum_{e \in \Gamma_{12}} \int_{e} p_{2}\left(\boldsymbol{v}_{1} \cdot \boldsymbol{n}_{12}\right)+\frac{\mu}{G} \sum_{e \in \Gamma_{12}} \int_{e}\left(\boldsymbol{u}_{1} \cdot \boldsymbol{\tau}_{12}\right)\left(\boldsymbol{v}_{1} \cdot \boldsymbol{\tau}_{12}\right) .
\end{gathered}
$$

The continuity of $\boldsymbol{u}_{1}$, the boundary condition (5), and Eqs. (34) and (35) yield:

$$
\mu a_{1}\left(\boldsymbol{u}_{1}, \boldsymbol{v}_{1}\right)+b\left(\boldsymbol{v}_{1}, p_{1}\right)+\Lambda\left(p_{2}, \boldsymbol{v}_{1}\right)=\left(\boldsymbol{f}_{1}, \boldsymbol{v}_{1}\right) .
$$

Equation (31) is a consequence of (2), (5) and the fact that $[\boldsymbol{u}] \cdot \boldsymbol{n}_{e}=0$ on each edge $e$. Now for the single phase flow part, we repeat the process with (4): multiply by a test function $q_{2}$, and integrate by parts and sum over all elements in $\mathcal{E}_{h}^{2}$. Definition (3) of $\boldsymbol{u}_{2}$, the regularity of $p_{2}$ and the boundary condition (6) give

$$
\begin{aligned}
\sum_{E \in \mathcal{E}_{h}^{2}} & \int_{E} \boldsymbol{K} \nabla p_{2} \cdot \nabla q_{2}-\sum_{e \in \Gamma_{h}^{2} \cup \Gamma_{12}} \int_{e}\left\{\boldsymbol{K} \nabla p_{2} \cdot \boldsymbol{n}_{e}\right\}\left[q_{2}\right] \\
& +\epsilon_{2} \sum_{e \in \Gamma_{h}^{2}} \int_{e}\left\{\boldsymbol{K} \nabla q_{2} \cdot \boldsymbol{n}_{e}\right\} \cdot\left[p_{2}\right]+\sum_{e \in \Gamma_{h}^{2}} \frac{\sigma_{e}}{|e|} \int_{e}\left[p_{2}\right]\left[q_{2}\right] \\
& -\sum_{e \in \Gamma_{12}} \int_{e}\left(\boldsymbol{u}_{2} \cdot \boldsymbol{n}_{12}\right) q_{2}=\left(f_{2}, q_{2}\right) .
\end{aligned}
$$

Using the interface condition (8) in the equation above, gives (32). Finally, Eq. (33) is just condition (7).

The discrete scheme is: Find $\left(\boldsymbol{U}_{1}, P_{1}, P_{2}\right) \in \boldsymbol{X}_{h}^{1} \times M_{h}^{1} \times M_{h}^{2}$ such that

$$
\begin{array}{rr}
\mu a_{1}\left(\boldsymbol{U}_{1}, \boldsymbol{v}_{1}\right)+b\left(\boldsymbol{v}_{1}, P_{1}\right)+\Lambda\left(P_{2}, \boldsymbol{v}_{1}\right)=\left(\boldsymbol{f}_{1}, \boldsymbol{v}_{1}\right), & \forall \boldsymbol{v}_{1} \in \boldsymbol{X}_{h}^{1}, \\
b\left(\boldsymbol{U}_{1}, q_{1}\right)=0, & \forall q_{1} \in M_{h}^{1}, \\
a_{2}\left(P_{2}, q_{2}\right)-\Lambda\left(q_{2}, \boldsymbol{U}_{1}\right)=\left(f_{2}, q_{2}\right), & \forall q_{2} \in M_{h}^{2}, \\
\int_{\Omega_{1}} P_{1}+\int_{\Omega_{2}} P_{2}=0 .
\end{array}
$$

Before addressing the existence and uniqueness of a solution to the numerical scheme, we recall the fact that the bilinear forms $a_{1}$ and $a_{2}$ are coercive with respect to $\|\cdot\|_{X^{1}}$ and $\|\cdot\|_{M^{2}}$, respectively. 
Lemma 3.2. Under Hypothesis A, there exist two positive constants $C_{1}$ and $C_{2}$ such that

$$
\begin{array}{ll}
C_{1}\left\|\boldsymbol{v}_{1}\right\|_{X^{1}}^{2} \leqslant a_{1}\left(\boldsymbol{v}_{1}, \boldsymbol{v}_{1}\right), & \forall \boldsymbol{v}_{1} \in \boldsymbol{X}_{h}^{1}, \\
C_{2}\left\|q_{2}\right\|_{M^{2}}^{2} \leqslant a_{2}\left(q_{2}, q_{2}\right), & \forall q_{2} \in M_{h}^{2} .
\end{array}
$$

Proof. Inequality (40) is easily derived from the Korn's inequality (1.19) in [5] for piecewise $H^{1}$ functions, obtained by Brenner. The proof of (41) is straightforward for the nonsymmetric bilinear forms, and is standard for the symmetric bilinear forms (Wheeler [22]).

Lemma 3.3. The discrete scheme has a unique solution.

Proof. Since (36)-(39) is a square system of linear equations in finite dimension, it suffices to prove that $\left(\boldsymbol{f}_{1}, f_{2}\right)=(\mathbf{0}, 0)$ implies $\left(\boldsymbol{U}_{1}, P_{1}, P_{2}\right)=(\mathbf{0}, 0,0)$. We choose $\boldsymbol{v}_{1}=\boldsymbol{U}_{1}$ in (36), $q_{1}=P_{1}$ in (37) and $q_{2}=$ $P_{2}$ in (38). Adding the resulting equations gives

$$
\mu a_{1}\left(\boldsymbol{U}_{1}, \boldsymbol{U}_{1}\right)+a_{2}\left(P_{2}, P_{2}\right)=0 .
$$

From Lemma 3.2, this implies $\left\|\boldsymbol{U}_{1}\right\|_{X^{1}}=\left\|P_{2}\right\|_{M^{2}}=0$, which means that $\boldsymbol{U}_{1}=0$ and $P_{2}$ is a global constant over $\Omega_{2}$. Equation (36), with (39) becomes

$$
b\left(\boldsymbol{v}_{1}, P_{1}\right)-\frac{1}{\left|\Omega_{2}\right|}\left(\int_{\Omega_{1}} P_{1}\right) \sum_{e \in \Gamma_{12}} \int_{e} \boldsymbol{v}_{1} \cdot \boldsymbol{n}_{12}=0, \quad \forall \boldsymbol{v}_{1} \in \boldsymbol{X}_{h}^{1} .
$$

We now write $P_{1}=\bar{P}_{1}+\tilde{P}_{1}$, where $\bar{P}_{1}=\left(1 /\left|\Omega_{1}\right|\right) \int_{\Omega_{1}} P_{1}$. The equation above becomes

$$
b\left(\boldsymbol{v}_{1}, \tilde{P}_{1}\right)+b\left(\boldsymbol{v}_{1}, \bar{P}_{1}\right)-\frac{\left|\Omega_{1}\right|}{\left|\Omega_{2}\right|} \bar{P}_{1} \sum_{e \in \Gamma_{12}} \int_{e} \boldsymbol{v}_{1} \cdot \boldsymbol{n}_{12}=0, \quad \forall \boldsymbol{v}_{1} \in \boldsymbol{X}_{h}^{1} .
$$

Note that

$$
b\left(\boldsymbol{v}_{1}, \bar{P}_{1}\right)=-\bar{P}_{1} \sum_{e \in \Gamma_{12}} \int_{e} \boldsymbol{v}_{1} \cdot \boldsymbol{n}_{12}
$$

So that, we have

$$
b\left(\boldsymbol{v}_{1}, \tilde{P}_{1}\right)-\left(1+\frac{\left|\Omega_{1}\right|}{\left|\Omega_{2}\right|}\right) \bar{P}_{1} \sum_{e \in \Gamma_{12}} \int_{e} \boldsymbol{v}_{1} \cdot \boldsymbol{n}_{12}=0, \quad \forall \boldsymbol{v}_{1} \in \boldsymbol{X}_{h}^{1} .
$$


Since $\tilde{P}_{1}$ belongs to $L_{0}^{2}\left(\Omega_{1}\right)$ and the spaces $H_{0}^{1}\left(\Omega_{1}\right)^{2}, L_{0}^{2}\left(\Omega_{1}\right)$ satisfy the exact inf-sup condition (see for example [13]), there exists $\tilde{\boldsymbol{v}} \in H_{0}^{1}\left(\Omega_{1}\right)^{2}$ such that $-\nabla \cdot \tilde{\boldsymbol{v}}=\tilde{P}_{1}$. Choose $\boldsymbol{v}_{1}=R_{h}(\tilde{\boldsymbol{v}})$ in (42), then we have from properties (14)-(16)

$$
\left\|\tilde{P}_{1}\right\|_{0, \Omega_{1}}^{2}=0 \text {. }
$$

This implies that $\tilde{P}_{1}=0$, and from (42), we have that $\bar{P}_{1}=0$, which also means that $P_{2}=0$.

Remark . The approximation $\boldsymbol{U}_{2}$ of the Darcy velocity $\boldsymbol{u}_{2}$ is a discontinuous piecewise polynomial vector of degree $k_{2}-1$ and is directly obtained from the discontinuous approximation $P_{2}$, by the formula $\boldsymbol{U}_{2}=$ $-K \nabla P_{2}$.

\section{ERROR ESTIMATES}

In this section, we derive first optimal error estimates in the energy norm for the Stokes velocity and in the $L^{2}$ norm for the Darcy pressure. A second estimate gives an optimal convergence rate for the $L^{2}$ norm of the pressure in the Stokes region.

Theorem 4.1. Let $(\boldsymbol{u}, p)$ be the solution of the coupled problem (1)(4), such that $\left.\boldsymbol{u}\right|_{\Omega_{1}} \in H^{k_{1}+1}\left(\Omega_{1}\right),\left.p\right|_{\Omega_{1}} \in H^{k_{1}}\left(\Omega_{1}\right)$ and $\left.p\right|_{\Omega_{2}} \in H^{k_{2}+1}\left(\Omega_{2}\right)$. The discrete solution $(\boldsymbol{U}, P)$ of (36)-(39) satisfies the error estimate:

$$
\begin{aligned}
\left\|\boldsymbol{u}_{1}-\boldsymbol{U}_{1}\right\|_{X^{1}}+\left\|p_{2}-P_{2}\right\|_{M^{2}} & \leqslant C h_{1}^{k_{1}}\left(\left|\boldsymbol{u}_{1}\right|_{k_{1}+1, \Omega_{1}}+|p|_{k_{1}, \Omega_{1}}\right) \\
& +C h_{2}^{k_{2}}\left(1+h_{1}^{-1 / 2} h_{2}^{1 / 2}\right)\left|p_{2}\right|_{k_{2}+1, \Omega_{2}},
\end{aligned}
$$

where $C$ is a constant independent of $h_{1}$ and $h_{2}$.

Proof. Let $\tilde{\boldsymbol{u}}_{1}=R_{h}\left(\boldsymbol{u}_{1}\right), \tilde{p}_{1}=r_{h}\left(p_{1}\right)$ and $\tilde{p}_{2}=r_{h}\left(p_{2}\right)$ be interpolants of $\boldsymbol{u}_{1}, p_{1}$ and $p_{2}$, respectively. Define $\chi=\boldsymbol{U}_{1}-\tilde{\boldsymbol{u}}_{1}$ and $\xi=P_{2}-\tilde{p}_{2}$. From (30)-(32) and (36)-(38), the error equations are

$$
\begin{aligned}
\mu a_{1}\left(\chi, \boldsymbol{v}_{1}\right)+b\left(\boldsymbol{v}_{1}, P_{1}-\tilde{p}_{1}\right)+\Lambda\left(\xi, \boldsymbol{v}_{1}\right)= & \mu a_{1}\left(\boldsymbol{u}_{1}-\tilde{\boldsymbol{u}}_{1}, \boldsymbol{v}_{1}\right) \\
& +b\left(\boldsymbol{v}_{1}, p_{1}-\tilde{p}_{1}\right)+\Lambda\left(p_{2}-\tilde{p}_{2}, \boldsymbol{v}_{1}\right), \\
& \forall \boldsymbol{v}_{1} \in X_{h}^{1}, \\
b\left(\chi, q_{1}\right)= & b\left(\boldsymbol{u}_{1}-\tilde{\boldsymbol{u}}_{1}, q_{1}\right), \quad \forall q_{1} \in M_{h}^{1}, \\
a_{2}\left(\xi, q_{2}\right)-\Lambda\left(q_{2}, \boldsymbol{\chi}\right)= & a_{2}\left(p_{2}-\tilde{p}_{2}, q_{2}\right)-\Lambda\left(q_{2}, \boldsymbol{u}_{1}-\tilde{\boldsymbol{u}}_{1}\right), \\
& \forall q_{2} \in M_{h}^{2} .
\end{aligned}
$$


Choosing $\boldsymbol{v}_{1}=\chi$ in (43), $q_{1}=P_{1}-\tilde{p}_{1}$ in (44), $q_{2}=\xi$ in (45) and summing the resulting equations gives

$$
\begin{aligned}
\mu a_{1}(\boldsymbol{\chi}, \boldsymbol{\chi})+a_{2}(\xi, \xi)= & \mu a_{1}\left(\boldsymbol{u}_{1}-\tilde{\boldsymbol{u}}_{1}, \boldsymbol{\chi}\right)+a_{2}\left(p_{2}-\tilde{p}_{2}, \xi\right)+b\left(\boldsymbol{\chi}, p_{1}-\tilde{p}_{1}\right) \\
& -b\left(\boldsymbol{u}_{1}-\tilde{\boldsymbol{u}}_{1}, P_{1}-\tilde{p}_{1}\right)+\Lambda\left(p_{2}-\tilde{p}_{2}, \boldsymbol{\chi}\right) \\
& -\Lambda\left(\xi, \boldsymbol{u}_{1}-\tilde{\boldsymbol{u}}_{1}\right) .
\end{aligned}
$$

We now bound each of the terms on the right-hand side of (46). We first rewrite $a_{1}\left(\boldsymbol{u}_{1}-\tilde{\boldsymbol{u}}_{1}, \boldsymbol{\chi}\right)$

$$
\begin{aligned}
\mu a_{1}\left(\boldsymbol{u}_{1}-\tilde{\boldsymbol{u}}_{1}, \chi\right)= & 2 \mu \sum_{E \in \mathcal{E}_{h}^{1}} \int_{E} \boldsymbol{D}\left(\boldsymbol{u}_{1}-\tilde{\boldsymbol{u}}_{1}\right): \boldsymbol{D}(\chi) \\
& -2 \mu \sum_{e \in \Gamma_{h}^{1} \cup \Gamma_{1}} \int_{e}\left\{\boldsymbol{D}\left(\boldsymbol{u}_{1}-\tilde{\boldsymbol{u}}_{1}\right)\right\} \boldsymbol{n}_{e} \cdot[\chi] \\
& +2 \mu \varepsilon_{1} \sum_{e \in \Gamma_{h}^{1} \cup \Gamma_{1}} \int_{e}\{\boldsymbol{D}(\chi)\} \boldsymbol{n}_{e} \cdot\left[\boldsymbol{u}_{1}-\tilde{\boldsymbol{u}}_{1}\right] \\
& +\mu \sum_{e \in \Gamma_{h}^{1} \cup \Gamma_{1}} \frac{\sigma_{1, e}}{|e|} \int_{e}\left[\boldsymbol{u}_{1}-\tilde{\boldsymbol{u}}_{1}\right] \cdot[\chi] \\
& +\frac{\mu}{G} \sum_{e \in \Gamma_{12}} \int_{e}\left(\left(\boldsymbol{u}_{1}-\tilde{\boldsymbol{u}}_{1}\right) \cdot \boldsymbol{\tau}_{12}\right)\left(\chi \cdot \boldsymbol{\tau}_{12}\right) \\
& +\mu \sum_{e \in \Gamma_{1}} \int_{e}\left(\boldsymbol{u}_{1}-\tilde{\boldsymbol{u}}_{1}\right) \cdot \chi=T_{1}+\cdots+T_{6} .
\end{aligned}
$$

Using the Cauchy-Schwarz inequality, and the approximation result (19), we have

$$
\begin{aligned}
T_{1} & \leqslant C \mu \sum_{E \in \mathcal{E}_{h}^{1}}\left\|\nabla\left(\boldsymbol{u}_{1}-\tilde{\boldsymbol{u}}_{1}\right)\right\|_{0, E}\|\nabla \chi\|_{0, E} \leqslant \frac{\mu}{8}\|\nabla \chi\|_{0, \Omega_{1}}^{2}+C\left\|\nabla\left(\boldsymbol{u}_{1}-\tilde{\boldsymbol{u}}_{1}\right)\right\|_{0, \Omega_{1}}^{2} \\
& \leqslant \frac{\mu}{8}\|\nabla \chi\|_{0, \Omega_{1}}^{2}+C h_{1}^{2 k_{1}}|\boldsymbol{u}|_{k_{1}+1, \Omega_{1}}^{2} .
\end{aligned}
$$

Let $L_{h}(\boldsymbol{u})$ denote the standard Lagrange interpolant of degree $k_{1}$ defined in $\Omega_{1}$ and let us insert it in the second integral term. For $e$ a segment of 
$\Gamma_{h}^{1} \cup \Gamma_{1}$, we have

$$
\begin{aligned}
\int_{e}\left\{\boldsymbol{D}\left(\boldsymbol{u}_{1}-\tilde{\boldsymbol{u}}_{1}\right)\right\} \boldsymbol{n}_{e} \cdot[\chi]= & \int_{e}\left\{\boldsymbol{D}\left(\boldsymbol{u}_{1}-L_{h}\left(\boldsymbol{u}_{1}\right)\right)\right\} \boldsymbol{n}_{e} \cdot[\chi] \\
& +\int_{e}\left\{\boldsymbol{D}\left(L_{h}\left(\boldsymbol{u}_{1}\right)-\tilde{\boldsymbol{u}}_{1}\right)\right\} \boldsymbol{n}_{e} \cdot[\chi] .
\end{aligned}
$$

Expanding the first integral, we obtain from the trace inequality (24) and from the fact that the Lagrange interpolant satisfies the optimal approximation result (19)

$$
\begin{aligned}
\mu & \sum_{e \in \Gamma_{h}^{1} \cup \Gamma_{1}} \int_{e}\left\{\boldsymbol{D}\left(\boldsymbol{u}_{1}-L_{h}\left(\boldsymbol{u}_{1}\right)\right)\right\} \boldsymbol{n}_{e} \cdot[\chi] \\
\leqslant & \sum_{e \in \Gamma_{h}^{1} \cup \Gamma_{1}} \frac{\sigma_{1, e}^{1 / 2}}{|e|^{1 / 2}}\|[\chi]\|_{0, e} \frac{|e|^{1 / 2}}{\sigma_{1, e}^{1 / 2}}\left\|\left\{\boldsymbol{D}\left(\boldsymbol{u}_{1}-L_{h}\left(\boldsymbol{u}_{1}\right)\right)\right\} \boldsymbol{n}_{e}\right\|_{0 . e} \\
\leqslant & \frac{\mu}{16} \sum_{e \in \Gamma_{h}^{1} \cup \Gamma_{1}} \frac{\sigma_{1, e}}{|e|}\|[\chi]\|_{0, e}^{2}+C h_{1}^{2 k_{1}}\left|\boldsymbol{u}_{1}\right|_{k_{1}+1, \Omega_{1}}^{2} .
\end{aligned}
$$

Similarly, if we denote by $E_{e}^{12}$ the elements sharing the edge $e$, and we use the trace inequality (25), a triangle inequality and the approximation results, then we have

$$
\begin{aligned}
\mu \sum_{e \in \Gamma_{h}^{1} \cup \Gamma_{1}} \int_{e}\left\{\boldsymbol{D}\left(L_{h}\left(\boldsymbol{u}_{1}\right)-\tilde{\boldsymbol{u}_{1}}\right)\right\} \boldsymbol{n}_{e} \cdot[\chi] \leqslant & \frac{\mu}{16} \sum_{e \in \Gamma_{h}^{1} \cup \Gamma_{1}} \frac{\sigma_{1, e}}{|e|}\|[\chi]\|_{0, e}^{2} \\
& +\sum_{e \in \Gamma_{h}^{1} \cup \Gamma_{1}} \frac{|e|}{\sigma_{1, e}} h_{e}^{-1}\left|\tilde{\boldsymbol{u}}_{1}-L_{h}\left(\boldsymbol{u}_{1}\right)\right|_{1, E_{e}^{12}}^{2} \\
\leqslant & \frac{\mu}{16} \sum_{e \in \Gamma_{h}^{1} \cup \Gamma_{1}} \frac{\sigma_{1, e}}{|e|}\|[\chi]\|_{0, e}^{2} \\
& +C h_{1}^{2 k_{1}}\left|\boldsymbol{u}_{1}\right|_{k_{1}+1, \Omega_{1}}^{2} .
\end{aligned}
$$

Therefore,

$$
T_{2} \leqslant \frac{\mu}{8} \sum_{e \in \Gamma_{h}^{1} \cup \Gamma_{1}} \frac{\sigma_{1, e}}{|e|}\|[\chi]\|_{0, e}^{2}+C h_{1}^{2 k_{1}}\left|\boldsymbol{u}_{1}\right|_{k_{1}+1, \Omega_{1}}^{2}
$$

The third term vanishes because of the properties (15), (16) satisfied by $\tilde{\boldsymbol{u}}$.

$$
T_{3}=0 \text {. }
$$


Using the Cauchy-Schwarz inequality, the jump term $T_{4}$ is bounded by virtue of (23) and (19)

$$
\begin{aligned}
T_{4} & \leqslant \frac{\mu}{8} \sum_{e \in \Gamma_{h}^{1} \cup \Gamma_{1}} \frac{\sigma_{1, e}}{|e|}\|[\chi]\|_{0, e}^{2}+C \sum_{e \in \Gamma_{h}^{1} \cup \Gamma_{1}} \frac{\sigma_{e, 1}}{|e|}\left\|\left[\boldsymbol{u}_{1}-\tilde{\boldsymbol{u}}_{1}\right]\right\|_{0, e}^{2} \\
& \leqslant \frac{\mu}{8} \sum_{e \in \Gamma_{h}^{1} \cup \Gamma_{1}} \frac{\sigma_{e, 1}}{|e|}\|[\chi]\|_{0, e}^{2}+C h_{1}^{2 k_{1}}\left|\boldsymbol{u}_{1}\right|_{k+1, \Omega_{1}}^{2} .
\end{aligned}
$$

The fifth term is bounded using the trace inequality (23) and the bound (19)

$$
\begin{aligned}
T_{5} & \leqslant \frac{\mu}{G} \sum_{e \in \Gamma_{1}}\left\|\boldsymbol{u}_{1}-\tilde{\boldsymbol{u}}_{1}\right\|_{0, e}\left\|\chi \cdot \boldsymbol{\tau}_{12}\right\|_{0, e} \\
& \leqslant \frac{\mu}{2 G} \sum_{e \in \Gamma_{12}}\left\|\chi \cdot \boldsymbol{\tau}_{12}\right\|_{0, e}^{2}+C \sum_{e \in \Gamma_{1}}\left(h_{e}^{-1}\left\|\boldsymbol{u}_{1}-\tilde{\boldsymbol{u}}_{1}\right\|_{0, E}^{2}+h_{e}\left|\boldsymbol{u}_{1}-\tilde{\boldsymbol{u}}_{1}\right|_{1, E}^{2}\right) \\
& \leqslant \frac{\mu}{2 G} \sum_{e \in \Gamma_{12}}\left\|\chi \cdot \boldsymbol{\tau}_{12}\right\|_{0, e}^{2}+C h_{1}^{2 k_{1}}\left|\boldsymbol{u}_{1}\right|_{k_{1}+1, \Omega_{1}}^{2}
\end{aligned}
$$

Finally the last term is bounded using the property (19) and (22).

$$
\begin{aligned}
T_{6} & \leqslant C h_{1}^{k_{1}+1 / 2}\left|\boldsymbol{u}_{1}\right|_{k_{1}+1, \Omega_{1}} h_{1}^{-1 / 2}\|\chi\|_{0, \Omega_{1}} \\
& \leqslant C h_{1}^{k_{1}}\left|\boldsymbol{u}_{1}\right|_{k_{1}+1, \Omega_{1}}\|\chi\|_{X^{1}} \\
& \leqslant \frac{\mu}{8}\|\boldsymbol{\chi}\|_{X^{1}}+C h_{1}^{2 k_{1}}\left|\boldsymbol{u}_{1}\right|_{k_{1}+1, \Omega_{1}}^{2} .
\end{aligned}
$$

Combining (47)-(52), we have

$$
\begin{aligned}
a_{1}\left(\boldsymbol{u}_{1}-\tilde{\boldsymbol{u}}_{1}, \chi\right) \leqslant & \frac{3 \mu}{4}\|\nabla \chi\|_{0, \Omega_{1}}^{2}+\frac{3 \mu}{4} \sum_{e \in \Gamma_{h}^{1} \cup \Gamma_{1}} \frac{\sigma_{1, e}}{|e|}\|[\chi]\|_{0, e}^{2} \\
& +\frac{\mu}{2 G} \sum_{e \in \Gamma_{12}}\left\|\chi \cdot \boldsymbol{\tau}_{12}\right\|_{0, e}^{2}+C h_{1}^{2 k_{1}}|\boldsymbol{u}|_{k_{1}+1, \Omega_{1}}^{2} .
\end{aligned}
$$


Let us now expand $a_{2}\left(p_{2}-\tilde{p}_{2}, \xi\right)$.

$$
\begin{aligned}
a_{2}\left(p_{2}-\tilde{p}_{2}, \xi\right)= & \sum_{E \in \mathcal{E}_{h}^{2}} \int_{E} \boldsymbol{K} \nabla\left(p_{2}-\tilde{p}_{2}\right) \cdot \nabla \xi+\sum_{e \in \Gamma_{h}^{2}} \frac{\sigma_{2, e}}{|e|} \int_{e}\left[p_{2}-\tilde{p}_{2}\right][\xi] \\
& -\sum_{e \in \Gamma_{h}^{2}} \int_{e}\left\{\boldsymbol{K} \nabla\left(p_{2}-\tilde{p}_{2}\right) \cdot \boldsymbol{n}_{e}\right\}[\xi] \\
& +\epsilon_{2} \sum_{e \in \Gamma_{h}^{2}} \int_{e}\left\{\boldsymbol{K} \nabla \xi \cdot \boldsymbol{n}_{e}\right\}\left[p_{2}-\tilde{p}_{2}\right] .
\end{aligned}
$$

Clearly, these terms are bounded in a similar fashion as the terms $T_{1}, \ldots, T_{4}$, using in particular the approximation result (21).

$$
a_{2}\left(p_{2}-\tilde{p}_{2}, \xi\right) \leqslant \frac{1}{8}\|\nabla \xi\|_{0, \Omega_{2}}^{2}+\frac{3}{4} \sum_{e \in \Gamma_{h}^{2}} \frac{\sigma_{2, e}}{|e|}\|[\xi]\|_{0, e}^{2}+C h_{2}^{2 k_{2}}\left|p_{2}\right|_{k_{2}+1, \Omega_{2}}^{2} .
$$

Using property (20) of the operator $r_{h}$, the third term on the right-hand side of (46) reduces to

$$
b\left(\chi, p_{1}-\tilde{p}_{1}\right)=\sum_{e \in \Gamma_{h}^{1} \cup \Gamma_{1}} \int_{e}\left\{p_{1}-\tilde{p}_{1}\right\}[\chi] \cdot \boldsymbol{n}_{e},
$$

which is bounded using the Cauchy-Schwarz inequality, trace inequality (23) and the estimate (21) by

$$
b\left(\chi, p_{1}-\tilde{p}_{1}\right) \leqslant \frac{\mu}{16} \sum_{e \in \Gamma_{h}^{1} \cup \Gamma_{1}} \frac{\sigma_{1, e}}{|e|}\|[\chi]\|_{0, e}^{2}+C h_{1}^{2 k_{1}}|p|_{k_{1}, \Omega_{1}}^{2} .
$$

The term $b\left(\boldsymbol{u}_{1}-\tilde{\boldsymbol{u}}_{1}, P_{1}-\tilde{p}_{1}\right)$ vanishes because of the properties (14)-(16) of $\tilde{\boldsymbol{u}}_{1}$. It suffices then to bound the interface terms $\Lambda\left(p_{2}-\tilde{p}_{2}, \boldsymbol{\chi}\right)$ and $\Lambda\left(\xi, \boldsymbol{u}_{1}-\tilde{\boldsymbol{u}}_{1}\right)$ in (46). Using the trace inequality (23), the approximation result (21) and the bound (22), we obtain

$$
\begin{aligned}
\Lambda\left(p_{2}-\tilde{p}_{2}, \chi\right) & \leqslant \sum_{e \in \Gamma_{12}}\left\|p_{2}-\tilde{p}_{2}\right\|_{0, e}\|\chi\|_{0, e} \\
& \leqslant C\|\chi\|_{0, \Omega_{1}} h_{2}^{k_{2}+1 / 2} h_{1}^{-1 / 2}\left|p_{2}\right|_{k_{2}+1, \Omega_{2}} \\
& \leqslant \frac{\mu}{16}\|\chi\|_{X^{1}}^{2}+C h_{2}^{2 k_{2}+1} h_{1}^{-1}\left|p_{2}\right|_{k_{2}+1, \Omega_{2}}^{2} .
\end{aligned}
$$


We now associate for each edge $e=\Gamma_{12} \cap \partial E^{1}$, for some $E^{1} \in \mathcal{E}_{h}^{1}$, the constant $c_{e}=(1 /|e|) \int_{e} \xi$. From the property (17) and (19), we can write

$$
\begin{aligned}
\Lambda\left(\xi, \boldsymbol{u}_{1}-\tilde{\boldsymbol{u}}_{1}\right) & =\sum_{e \in \Gamma_{12}} \int_{e}\left(\xi-c_{e}\right)\left(\boldsymbol{u}_{1}-\tilde{\boldsymbol{u}}_{1}\right) \cdot \boldsymbol{n}_{12} \\
& \leqslant \sum_{e \in \Gamma_{12}}\left\|\xi-c_{e}\right\|_{0, e}\left\|\boldsymbol{u}_{1}-\tilde{\boldsymbol{u}}_{1}\right\|_{0, e} \\
& \leqslant C h_{1}^{k_{1}+1 / 2} h_{2}^{1 / 2}\|\nabla \xi\|_{0, \Omega_{2}}\left|\boldsymbol{u}_{1}\right|_{k_{1}+1, \Omega_{1}} \\
& \leqslant \frac{1}{8}\|\xi\|_{M^{2}}^{2}+C h_{1}^{2 k_{1}}\left|\boldsymbol{u}_{1}\right|_{k_{1}+1, \Omega_{1}}^{2} .
\end{aligned}
$$

Collecting the bounds (53)-(57) and using Lemma 3.2 yields

$$
\begin{aligned}
\|\chi\|_{X^{1}}^{2}+\|\xi\|_{M^{2}}^{2} \leqslant & C h_{1}^{2 k_{1}}\left(\left|\boldsymbol{u}_{1}\right|_{k_{1}+1, \Omega_{1}}^{2}+\left|p_{1}\right|_{k_{1}, \Omega_{1}}^{2}\right) \\
& +C h_{2}^{2 k_{2}}\left(1+h_{1}^{-1} h_{2}\right)\left|p_{2}\right|_{k_{2}+1, \Omega_{2}}^{2} .
\end{aligned}
$$

The final result is obtained with a triangle inequality and approximation results.

Remark . Clearly, Theorem 4.1 gives an estimate of the error in the $L^{2}$ norm of the Darcy velocity. The convergence rate is optimal.

Theorem 4.2. Under the assumptions and notation of Theorem 4.1, we have

$$
\begin{aligned}
\left\|p_{1}-P_{1}\right\|_{0, \Omega_{1}} \leqslant & C h_{1}^{k_{1}}\left(\left|\boldsymbol{u}_{1}\right|_{k_{1}+1, \Omega_{1}}+\left|p_{1}\right|_{k_{1}, \Omega_{1}}\right) \\
& +C h_{2}^{k_{2}}\left(1+h_{1}^{-1 / 2} h_{2}^{1 / 2}\right)\left|p_{2}\right|_{k_{2}+1, \Omega_{2}} .
\end{aligned}
$$

where $C$ is a constant independent of $h_{1}$ and $h_{2}$.

Proof. Subtracting (30) from (36) gives

$$
\mu a_{1}\left(\boldsymbol{U}_{1}-\boldsymbol{u}_{1}, \boldsymbol{v}_{1}\right)+b\left(\boldsymbol{v}_{1}, P_{1}-p_{1}\right)+\Lambda\left(P_{2}-p_{2}, \boldsymbol{v}_{1}\right)=0, \quad \forall \boldsymbol{v}_{1} \in \boldsymbol{X}_{h}^{1} .
$$

As in Theorem 4.1, let $\tilde{p}_{1}=r_{h}\left(p_{1}\right), \tilde{p}_{2}=r_{h}\left(p_{2}\right), \zeta=P_{1}-\tilde{p}_{1}$ and $\xi=P_{2}-$ $\tilde{p}_{2}$. The error equation becomes

$$
\begin{aligned}
-b\left(\boldsymbol{v}_{1}, \zeta\right)= & \mu a_{1}\left(\boldsymbol{U}_{1}-\boldsymbol{u}_{1}, \boldsymbol{v}_{1}\right)+\Lambda\left(\xi, \boldsymbol{v}_{1}\right) \\
& -\Lambda\left(p_{2}-\tilde{p}_{2}, \boldsymbol{v}_{1}\right)-b\left(\boldsymbol{v}_{1}, p_{1}-\tilde{p}_{1}\right), \quad \forall \boldsymbol{v}_{1} \in \boldsymbol{X}_{h}^{1} .
\end{aligned}
$$


If we decompose $\zeta=\bar{\zeta}+\tilde{\zeta}$, where $\bar{\zeta}=\left(1 /\left|\Omega_{1}\right|\right) \int_{\Omega_{1}} \zeta$, and $\xi=\bar{\xi}+\tilde{\xi}$, where $\bar{\xi}=\left(1 /\left|\Omega_{2}\right|\right) \int_{\Omega_{2}} \xi$, then (39) and (33) with property (20) yield

$$
\left|\Omega_{1}\right| \bar{\zeta}+\left|\Omega_{2}\right| \bar{\xi}=0
$$

The error equation is then rewritten

$$
\begin{aligned}
-b\left(\boldsymbol{v}_{1}, \tilde{\zeta}\right)+\left(1+\frac{\left|\Omega_{1}\right|}{\left|\Omega_{2}\right|}\right) \bar{\zeta} \sum_{e \in \Gamma_{12}} \int_{e} \boldsymbol{v}_{1} \cdot \boldsymbol{n}_{12}= & \mu a_{1}\left(\boldsymbol{U}_{1}-\boldsymbol{u}_{1}, \boldsymbol{v}_{1}\right) \\
& +\Lambda\left(\tilde{\xi}, \boldsymbol{v}_{1}\right)-\Lambda\left(p_{2}-\tilde{p}_{2}, \boldsymbol{v}_{1}\right) \\
& -b\left(\boldsymbol{v}_{1}, p_{1}-\tilde{p}_{1}\right), \forall \boldsymbol{v}_{1} \in \boldsymbol{X}_{h}^{1}
\end{aligned}
$$

Since $\tilde{\zeta}$ belongs to $L_{0}^{2}\left(\Omega_{1}\right)$, there exists $\tilde{\boldsymbol{v}} \in H_{0}^{1}\left(\Omega_{1}\right)^{2}$ such that $-\nabla \cdot \tilde{\boldsymbol{v}}=\tilde{\zeta}$ and $|\tilde{\boldsymbol{v}}|_{1, \Omega_{1}} \leqslant C\|\tilde{\zeta}\|_{0, \Omega_{1}}$. Choose $\boldsymbol{v}_{1}=R_{h}(\tilde{\boldsymbol{v}})$ in (58). From properties (14)(16), (58) is reduced to

$$
\begin{aligned}
\|\tilde{\zeta}\|_{0, \Omega_{1}}^{2}= & \mu a_{1}\left(\boldsymbol{U}_{1}-\boldsymbol{u}_{1}, \boldsymbol{v}_{1}\right)+\Lambda\left(\tilde{\xi}, \boldsymbol{v}_{1}\right)-\Lambda\left(p_{2}-\tilde{p}_{2}, \boldsymbol{v}_{1}\right) \\
& -b\left(\boldsymbol{v}_{1}, p_{1}-\tilde{p}_{1}\right) .
\end{aligned}
$$

We expand and bound each term on the right-hand side of (59).

$$
\begin{aligned}
a\left(\boldsymbol{U}_{1}-\boldsymbol{u}_{1}, \boldsymbol{v}_{1}\right)= & 2 \mu \sum_{E \in \mathcal{E}_{h}^{1}} \int_{E} \boldsymbol{D}\left(\boldsymbol{U}_{1}-\boldsymbol{u}_{1}\right): \boldsymbol{D}\left(\boldsymbol{v}_{1}\right) \\
& +\sum_{e \in \Gamma_{h}^{1} \cup \Gamma_{1}} \frac{\sigma_{1, e}}{|e|} \int_{e}\left[\boldsymbol{U}_{1}-\boldsymbol{u}_{1}\right] \cdot\left[\boldsymbol{v}_{1}\right] \\
& -2 \mu \sum_{e \in \Gamma_{h}^{1} \cup \Gamma_{1}} \int_{e}\left\{\boldsymbol{D}\left(\boldsymbol{U}_{1}-\boldsymbol{u}_{1}\right) \boldsymbol{n}_{e}\right\} \cdot\left[\boldsymbol{v}_{1}\right] \\
& +2 \mu \epsilon \sum_{e \in \Gamma_{h}^{1} \cup \Gamma_{1}} \int_{e}\left\{\boldsymbol{D}\left(\boldsymbol{v}_{1}\right) \boldsymbol{n}_{e}\right\} \cdot\left[\boldsymbol{U}_{1}-\boldsymbol{u}_{1}\right] \\
& +\frac{\mu}{G} \sum_{e \in \Gamma_{12}} \int_{e}\left(\boldsymbol{U}_{1}-\boldsymbol{u}_{1}\right) \cdot \boldsymbol{\tau}_{12}\left(\boldsymbol{v}_{1} \cdot \boldsymbol{\tau}_{12}\right)+\sum_{e \in \Gamma_{1}} \int_{e}\left(\boldsymbol{U}_{1}-\boldsymbol{u}_{1}\right) \cdot \boldsymbol{v}_{1} \\
= & Q_{1}+\cdots+Q_{6} .
\end{aligned}
$$

The bounds for $Q_{1}, Q_{2}, Q_{4}, Q_{5}$ are easily obtained

$$
Q_{1}+Q_{2}+Q_{4}+Q_{5} \leqslant C\left\|\boldsymbol{U}_{1}-\boldsymbol{u}_{1}\right\|_{X^{1}}\left\|\boldsymbol{v}_{1}\right\|_{X^{1}} .
$$


The remaining terms are bounded by introducing the interpolant $\tilde{\boldsymbol{u}}_{1}=$ $R_{h}\left(\boldsymbol{u}_{1}\right)$.

$$
\begin{array}{r}
Q_{3}=-2 \mu \sum_{e \in \Gamma_{h}^{1} \cup \Gamma_{1}} \int_{e}\left\{\boldsymbol{D}\left(\boldsymbol{U}_{1}-\tilde{\boldsymbol{u}}_{1}\right) \boldsymbol{n}_{e}\right\} \cdot\left[\boldsymbol{v}_{1}\right] \\
-2 \mu \sum_{e \in \Gamma_{h}^{1} \cup \Gamma_{1}} \int_{e}\left\{\boldsymbol{D}\left(\tilde{\boldsymbol{u}}_{1}-\boldsymbol{u}_{1}\right) \boldsymbol{n}_{e}\right\} \cdot\left[\boldsymbol{v}_{1}\right] .
\end{array}
$$

The first part vanishes because of (15) and (16) and the second part is bounded like $T_{2}$ in Theorem 4.1. Therefore, we have

$$
a_{1}\left(\boldsymbol{U}_{1}-\boldsymbol{u}_{1}, \boldsymbol{v}_{1}\right) \leqslant\left\|\boldsymbol{v}_{1}\right\|_{X^{1}}\left(\left\|\boldsymbol{U}_{1}-\boldsymbol{u}_{1}\right\|_{X^{1}}+C h_{1}^{k_{1}}\left|\boldsymbol{u}_{1}\right|_{k_{1}+1, \Omega_{1}}\right) .
$$

Similarly, from (17) and (19), we have

$$
\begin{aligned}
Q_{6}= & \sum_{e \in \Gamma_{1}} \int_{e}\left(\boldsymbol{U}_{1}-\tilde{\boldsymbol{u}}_{1}\right) \cdot \boldsymbol{v}_{1}+\sum_{e \in \Gamma_{1}} \int_{e}\left(\tilde{\boldsymbol{u}}_{1}-\boldsymbol{u}_{1}\right) \cdot \boldsymbol{v}_{1} \\
& \leqslant C\left(\left\|\boldsymbol{U}_{1}-\tilde{\boldsymbol{u}}_{1}\right\|_{0, \Omega_{1}}+h_{1}^{k_{1}}\left|\boldsymbol{u}_{1}\right|_{k_{1}+1, \Omega_{1}}\right)\left\|\boldsymbol{v}_{1}\right\|_{X^{1}} .
\end{aligned}
$$

Let us denote by $E_{e}$ the element in $\mathcal{E}_{h}^{2}$ that shares the edge $e$ of the interface $\Gamma_{12}$. Define also the constant $c_{e}=(1 /|e|) \int_{e} \tilde{\xi}$. From property (16) of the operator $R_{h}$, we have

$$
\begin{aligned}
\sum_{e \in \Gamma_{12}} \int_{e} \tilde{\xi} \boldsymbol{v}_{1} \cdot \boldsymbol{n}_{12} & =\sum_{e \in \Gamma_{12}} \int_{e}\left(\tilde{\xi}-c_{e}\right) \boldsymbol{v}_{1} \cdot \boldsymbol{n}_{12} \\
& \leqslant C \sum_{e \in \Gamma_{12}}|e|^{1 / 2}\|\nabla \tilde{\xi}\|_{0, E_{e}}\left\|\boldsymbol{v}_{1}\right\|_{0, e} \\
& \leqslant C h_{2}^{1 / 2}\|\nabla \xi\|_{0, \Omega_{2}}\left(\sum_{e \in \Gamma_{12}}\left\|\boldsymbol{v}_{1}\right\|_{0, e}^{2}\right)^{1 / 2} .
\end{aligned}
$$

By (21) and (23), we have

$$
\begin{aligned}
\sum_{e \in \Gamma_{12}} \int_{e}\left(p_{2}-\tilde{p}_{2}\right) \boldsymbol{v}_{1} \cdot \boldsymbol{n}_{12} & \leqslant \sum_{e \in \Gamma_{12}}\left\|p_{2}-\tilde{p}_{2}\right\|_{0, e}\left\|\boldsymbol{v}_{1}\right\|_{0, e} \\
& \leqslant C h_{2}^{k_{2}+1 / 2}\left|p_{2}\right|_{k_{2}+1, \Omega_{2}}\left(\sum_{e \in \Gamma_{12}}\left\|\boldsymbol{v}_{1}\right\|_{0, e}^{2}\right)^{1 / 2} .
\end{aligned}
$$


Now, since $\boldsymbol{v}_{1}$ satisfies $\int_{e} \boldsymbol{v}_{1}=0$ for any edge $e$ in $\Gamma_{12}$, we have

$$
\left(\sum_{e \in \Gamma_{12}}\left\|\boldsymbol{v}_{1}\right\|_{0, e}^{2}\right)^{1 / 2} \leqslant C h_{1}^{1 / 2}\left\|\nabla \boldsymbol{v}_{1}\right\|_{0, \Omega_{1}} \leqslant C h_{1}^{1 / 2}\left\|\boldsymbol{v}_{1}\right\|_{X^{1}} .
$$

Therefore, the resulting bound is

$$
\Lambda\left(\tilde{\xi}, \boldsymbol{v}_{1}\right)-\Lambda\left(p_{2}-\tilde{p}_{2}, \boldsymbol{v}_{1}\right) \leqslant C h_{1}^{1 / 2} h_{2}^{1 / 2}\left\|\boldsymbol{v}_{1}\right\|_{X^{1}} \times\left(\left\|P_{2}-\tilde{p}_{2}\right\|_{M^{2}}+h_{2}^{k_{2}}\left|p_{2}\right|_{k_{2}+1, \Omega_{2}}\right) .
$$

Finally, the bound for $b\left(v_{1}, p_{1}-\tilde{p}_{1}\right)$ is similar to the one obtained in (55)

$$
b\left(\boldsymbol{v}_{1}, p_{1}-\tilde{p}_{1}\right) \leqslant C\left\|\boldsymbol{v}_{1}\right\|_{X^{1}} h_{1}^{k_{1}}\left|p_{1}\right|_{k_{1}, \Omega_{1}} .
$$

From properties (19) and (18), we have

$$
\left\|\boldsymbol{v}_{1}\right\|_{X^{1}} \leqslant\left\|R_{h}(\tilde{\boldsymbol{v}})-\tilde{\boldsymbol{v}}\right\|_{X^{1}}+\|\tilde{\boldsymbol{v}}\|_{X^{1}} \leqslant C|\tilde{\boldsymbol{v}}|_{1, \Omega_{1}} \leqslant C\|\tilde{\zeta}\|_{0, \Omega_{1}} .
$$

Combining all the bounds above gives

$$
\begin{aligned}
\|\tilde{\zeta}\|_{0, \Omega_{1}} \leqslant & C\left\|\boldsymbol{U}_{1}-\boldsymbol{u}_{1}\right\|_{X^{1}}+C\left\|P_{2}-\tilde{p}_{2}\right\|_{M^{2}} \\
& +C h_{1}^{k_{1}}\left(\left|\boldsymbol{u}_{1}\right|_{k_{1}+1, \Omega_{1}}+\left|p_{1}\right|_{k_{1}, \Omega_{1}}\right)+C h_{2}^{k_{2}}\left|p_{2}\right|_{k_{2}+1, \Omega_{2}} .
\end{aligned}
$$

To finish the proof of the theorem, it remains to bound $\|\bar{\zeta}\|_{0, \Omega_{1}}$. This is accomplished by choosing a particular test function $\boldsymbol{v}_{1}$ in (58). Let $\rho$ be a function in $\mathcal{C}^{2}(\bar{\Omega})^{2}$, with compact support in $\Omega$ such that

$$
\sum_{e \in \Gamma_{12}} \int_{e} \boldsymbol{\rho} \cdot \boldsymbol{n}_{12}=1
$$

Denote $\overline{\boldsymbol{v}}=\left|\Omega_{1}\right| \bar{\zeta} \rho$ and choose $\boldsymbol{v}_{1}=R_{h}(\overline{\boldsymbol{v}})$. We first show that $\left\|\boldsymbol{v}_{1}\right\|_{X^{1}}$ is bounded by $\|\bar{\zeta}\|_{0, \Omega_{1}}$. By property (18) and (19), we have

$$
\left\|\boldsymbol{v}_{1}\right\|_{X^{1}} \leqslant\left\|R_{h}(\overline{\boldsymbol{v}})-\overline{\boldsymbol{v}}\right\|_{X^{1}}+\|\overline{\boldsymbol{v}}\|_{X^{1}} \leqslant C\|\nabla \overline{\boldsymbol{v}}\|_{0, \Omega_{1}}+\|\overline{\boldsymbol{v}}\|_{X^{1}} .
$$

But,

$$
\|\overline{\boldsymbol{v}}\|_{X^{1}}^{2}=\|\nabla \overline{\boldsymbol{v}}\|_{0, \Omega_{1}}^{2}+\frac{\left|\Omega_{1}\right|^{2} \bar{\zeta}^{2}}{G} \sum_{e \in \Gamma_{12}} \int_{e}\left(\boldsymbol{\rho} \cdot \boldsymbol{\tau}_{12}\right)^{2} \leqslant C\left|\Omega_{1}\right|\|\bar{\zeta}\|_{0, \Omega_{1}}^{2} .
$$

Thus,

$$
\left\|\boldsymbol{v}_{1}\right\|_{X^{1}} \leqslant C\|\bar{\zeta}\|_{0, \Omega_{1}}
$$


With that test function $v_{1}$, the error equation (58) becomes

$$
\begin{aligned}
\left(1+\frac{\left|\Omega_{1}\right|}{\left|\Omega_{2}\right|}\right)\|\bar{\zeta}\|_{0, \Omega_{1}}^{2}= & \mu a_{1}\left(\boldsymbol{U}_{1}-\boldsymbol{u}_{1}, \boldsymbol{v}_{1}\right) \\
& +\Lambda\left(\tilde{\xi}, \boldsymbol{v}_{1}\right)-\Lambda\left(p_{2}-\tilde{p}_{2}, \boldsymbol{v}_{1}\right) \\
& -b\left(\boldsymbol{v}_{1}, p_{1}-\tilde{p}_{1}\right)+b\left(\boldsymbol{v}_{1}, \tilde{\zeta}\right)
\end{aligned}
$$

Except for the last term, all the terms on the right-hand side of (62) are bounded exactly as in (59). We now rewrite the last term.

$$
b\left(\boldsymbol{v}_{1}, \tilde{\zeta}\right)=b\left(\tilde{\zeta}, R_{h}(\overline{\boldsymbol{v}})-\overline{\boldsymbol{v}}\right)+b(\overline{\boldsymbol{v}}, \tilde{\zeta})
$$

Clearly,

$$
b(\overline{\boldsymbol{v}}, \tilde{\zeta})=-\left|\Omega_{1}\right| \bar{\zeta} \sum_{E \in \mathcal{E}_{h}^{1}} \int_{E} \tilde{\zeta} \nabla \cdot \rho \leqslant C\left|\Omega_{1}\right|^{1 / 2}\|\bar{\zeta}\|_{0, \Omega_{1}}\|\tilde{\zeta}\|_{0, \Omega_{1}} .
$$

And it is easy to check from (14) to (16) that

$$
b\left(R_{h}(\overline{\boldsymbol{v}})-\overline{\boldsymbol{v}}, \tilde{\zeta}\right)=0
$$

Finally, we obtain from (62) and the bounds above

$$
\begin{aligned}
\|\bar{\zeta}\|_{0, \Omega_{1}} \leqslant & C\left\|\boldsymbol{U}_{1}-\boldsymbol{u}_{1}\right\|_{X^{1}}+C\left\|P_{2}-\tilde{p}_{2}\right\|_{M^{2}}+C\|\tilde{\zeta}\|_{0, \Omega_{1}} \\
& +C h_{1}^{k_{1}}\left(\left|\boldsymbol{u}_{1}\right|_{k_{1}+1, \Omega_{1}}+\left|p_{1}\right|_{k_{1}, \Omega_{1}}\right)+C h_{2}^{k_{2}}\left|p_{2}\right|_{k_{2}+1, \Omega_{2}} .
\end{aligned}
$$

The bounds (59), (63), (21) and Theorem 4.1 give the final result.

Remark. The results stated in Theorems 4.1 and 4.2 hold true in the case, where $k_{2} \geqslant 2$ and the parameter $\sigma_{2, e}$ is equal to zero, for all edges $e$ in $\Gamma_{h}^{1} \cup \Gamma^{1}$. In this case, the proof differs in the choice of the interpolant $\tilde{p}_{2}$, which now has to satisfy special flux properties (see [19] for further details).

\section{CONCLUSION}

In this paper, a discontinuous Galerkin method is formulated for the coupled Stokes and Darcy equations. Both symmetric and non-symmetric cases are considered. Optimal convergence rates are obtained for the fluid velocity and pressure. 


\section{ACKNOWLEDGMENTS}

The author wishes to thank Professor Ivan Yotov and the referees for their valuable comments.

\section{REFERENCES}

1. Adams, R. (1975). Sobolev Spaces, Academic Press, New York.

2. Arnold, D. N. (1982). An interior penalty finite element method with discontinuous elements. SIAM J. Numer. Anal. 19(4), 742-760.

3. Beavers, G. S., and Joseph, D. D. (1967). Boundary conditions at a naturally impermeable wall. J. Fluid. Mech. 30, 197-207.

4. Bernardi, C., Hecht, F., and Pironneau, O. (2002). Coupling Darcy and Stokes Equations for Porous Media with Cracks, Technical Report R02042, Paris VI.

5. Brenner, S. (2003). Korn's inequalities for piecewise $H^{1}$ vector fields Mathematics of Computation, S 0025-5718(03)01579-5, Article electronically published.

6. Crouzeix, M., and Falk, R. S. (1989). Non conforming finite elements for the Stokes problem. Math. of Comp. 52, 437-456.

7. Crouzeix, M., and Raviart, P.-A. (1973). Conforming and nonconforming finite element methods for solving the stationary Stokes equations. I. Rev. Française Automat. Informat. Recherche Opérationnelle Sér. Rouge. 7(R-3), 33-75.

8. Discacciati, M., Miglio, E., and Quarteroni, A. (2001). Mathematical and numerical models for coupling surface and groundwater flows. Appl. Numer. Math. 43(1-2), 57-74, 2002. 19th Dundee Biennial Conference on Numerical Analysis.

9. Discacciati, M., and Quarteroni, A. (2003). Analysis of a domain decomposition method for the coupling of stokes and darcy equations. In Brezzi et al. (eds.) Numerical Analysis and Advanced Applications - Enumath 2001, Springer, Milan, pp. 3-20.

10. Ewing, R. E., Iliev, O. P., and Lazarov, R. D. (1992). Numerical Simulation of Contamination Transport Due to Flow in Liquid and Porous Media, Technical Report 1992-10, Enhanced Oil Recovery Institute, University of Wyoming.

11. Fortin, M., and Soulié, M. (1983). A non-conforming piecewise quadratic finite element on triangles. Int. J. Nume. Methods Eng., 19, 505-520.

12. Girault, V., Rivière, B., and Wheeler, M. F. (2002). A Discontinuous Galerkin Method With Non-Overlapping Domain Decomposition for the Stokes and Navier-Stokes Problems, Technical Report TICAM 02-08, to appear in Mathematics of Computation.

13. Girault, V., and Raviart, P.-A. (1986). Finite element methods for Navier-Stokes Equations, Springer-Verlag, Berlin.

14. Jäger, W., and Mikelić, A. (2000). On the interface boundary condition of Beavers, Joseph, and Saffman. SIAM J. Appl. Math. 60, 1111-1127.

15. Layton, W. J., Schieweck, F., and Yotov, I. (2003). Coupling fluid flow with porous media flow. SIAM J. Numer. Anal. 40(6), 2195-2218.

16. Mardal, K.,E., Tai, X.-C., and Winther, R. (2002). A robust finite element method for Darcy-Stokes flow. SIAM J. Numer. Anal. 40(5), 1605-1631 (electronic).

17. Payne, L. E., and Straughan, B. (1998). Analysis of the boundary condition at the interface between a viscous fluid and a porous medium and related modelling questions. $J$. Math. Pures Appl. 77, 317-354.

18. Rivière, B., Wheeler, M. F., and Girault, V. (1999). Improved energy estimates for interior penalty, constrained and discontinuous Galerkin methods for elliptic problems. Part I. Comput. Geosci. 3, 337-360. 
19. Rivière, B., Wheeler, M. F., and Girault, V. (2001). A priori error estimates for finite element methods based on discontinuous approximation spaces for elliptic problems. SIAM J. Numer. Anal. 39, 902-931.

20. Rivière, B., and Yotov, I. (2003). Locally conservative coupling of Stokes and Darcy flow. SIAM J. Numer. Anal. accepted, 2004.

21. Saffman, P. (1971). On the boundary condition at the surface of a porous media. Stud. Appl. Math. 50, 292-315.

22. Wheeler, M. F. (1978). An elliptic collocation-finite element method with interior penalties. SIAM J. Numer. Anal. 15(1), 152-161. 